\title{
Prácticas docentes para sensibilizar socialmente al alumnado universitario: el caso de la asignatura de Organización de la Producción
}

Teaching practices for socially awareness of university students: the case of the subject of Production Management

Dolores Queiruga (dolores.queiruga@unirioja.es)

Universidad de La Rioja (España)

Javier González Benito (javiergb@usal.es)

Luz Amira Rocha Valencia (larocha@elpoli.edu.co)

Gustavo Lannelongue Nieto (lannelongue@usal.es)

Universidad de Salamanca (España)

http://dx.doi.org/10.12795/EDUCADE.2014.105.02

RESUMEN: Según muchos economistas, la crisis que estamos sufriendo no es sólo una crisis económica, sino una crisis de valores. La situación actual de producción y consumo es insostenible y requiere un cambio en las empresas y en la sociedad.

Hay algunas iniciativas empresariales y de consumidores que nos hacen pensar que el cambio de actitud en las personas y en el modelo empresarial se empieza a producir. Todas ellas hablan del valor de compartir, de la solidaridad, de la confianza y de la sostenibilidad.

En la Universidad, la Educación para el Desarrollo Sostenible (EDS) va cobrando cada vez mayor relevancia y va consolidándose. Sin embargo, estos temas no se suelen trasladar a los temarios de las asignaturas.

El objetivo de este trabajo es contribuir a la introducción de la sensibilización social de los estudiantes universitarios en el aula a través de dos trabajos prácticos realizados en la asignatura de Organización de la Producción, en Ingeniería Electrónica Industrial y Automática, en la Universidad de La Rioja. Estas actividades son descritas con la idea de que puedan ser transferidas a otras asignaturas y otras titulaciones.

Tras su puesta en práctica en el aula hemos podido comprobar que resultan muy motivadoras para los estudiantes porque tratan sobre la realidad de su entorno, les hacen valorar el esfuerzo de las personas, pensar en las necesidades sociales y en cómo ellos podrían ayudar. En una encuesta realizada al final del curso a los estudiantes, se les preguntó sobre la idoneidad de estas prácticas y prácticamente la totalidad de estudiantes las encontraron apropiadas y aprendieron con ellas. Las notas de los estudiantes fueron muy satisfactorias.

Creemos que este tipo de actividades puede contribuir a mejorar la actitud de nuestros estudiantes ante la sociedad y al mismo tiempo facilitarles la incorporación al mercado laboral.

PALABRAS CLAVE: Educación para el Desarrollo Sostenible, Sensibilización social, Prácticas sociales y solidarias, Organización de la Producción. 
ABSTRACT: According to many economists, the crisis we are experiencing is not only an economic crisis but a crisis of values.

The current situation of production and consumption is not sustainable and requires a change in business and society.

There are some businesses and consumer initiatives that make us think that the change of attitude in people and in the business model begins to occur. They all talk about the value of sharing, solidarity, trust and sustainability.

In Universities, Education for Sustainable Development (ESD) is gaining increasing relevance and strengthens. However, these issues are not usually included in the subjects.

The aim of this paper is to introduce the social awareness of university students in the classroom through two practices in the course of Production Management, in Industrial Electronics and Automation Engineering at the University of La Rioja. These activities are described with the idea that they can be transferred to other subjects and other university degrees. After its implementation in the classroom we have seen that are highly motivating for students because they deal with the reality of their environment, appreciate the efforts of people, think about social needs and how they could contribute to help.

In a survey conducted at the end of the course, students were asked about the adequacy of these practices and virtually all students found them appropriate and learned with them. The notes of the students were very satisfactory.

We believe that this type of activity can help to improve our students' attitude to society and at the same time facilitate labor market entry.

KEYWORDS: Education for Sustainable Development, Social awareness, Social and solidarity practices, Production Management.

\section{INTRODUCCIÓN}

Unos años antes de que comenzara la crisis actual, algunos autores advertían de que el modelo de desarrollo económico estaba agotado desde el punto de vista del bien de la humanidad y del deseo razonable de progreso de los países más pobres (Escámez et al., 2003). Asimismo, señalaban, que la situación de producción y consumo era insostenible y requería un cambio en las empresas y en la sociedad (Colom Cañellas, 2000). También advertían sobre los problemas éticos derivados de fomentar únicamente la maximización del consumo, la productividad y la ganancia (Gómez-Heras, 1997).

Ya en medio de la crisis, en el encuentro del Foro Económico Mundial de Davos del año 2012 fue recurrente el debate los valores y principios éticos y sobre el tema social y medioambiental y fueron considerados el primer punto de la agenda. Se sugirió la creación de un marco de valores, tomando como referencia el hecho de que, "según un reciente encuentro realizado para esta institución cubriendo 10 países y más de 130 mil personas, el $70 \%$ de los encuestados reconocieron que la crisis económica actual está vinculada a una crisis de principios y valores éticos que siembra serias dudas sobre la sustentabilidad del proceso de la globalización actual" (Moreno, 2013).

Igualmente, otros economistas opinan que estamos siendo testigos de una profunda crisis que no es solamente económico-financiera, sino sobre todo, una crisis de humanidad, constatando que el desequilibrio humano, social y ambiental ha sido provocado por la insaciable ambición de acumular riqueza y la incontrolada explotación de los recursos naturales (Bermúdez López, 2010). Por tanto, la crisis que estamos viviendo sería la consecuencia última de una sociedad acostumbrada a 
satisfacciones inmediatas, a la falta de responsabilidad, y a otras actitudes como el individualismo, materialismo o relativismo (Sánchez-Quiñones, 2011; Sánchez Cámara, 2012).

Está claro, por tanto, que vivimos en un momento en el que es absolutamente necesario modificar los valores sobre los que hemos diseñado nuestro modelo de convivencia y crear nuevos modelos (Sánchez Cámara, 2012). Para ello, es necesario que la sociedad y sus empresas estén cimentadas en el valor de las personas (Rovira y Olmos, 2013). De esta forma, como afirma Méndez García (2012), una economía que merezca el calificativo de humana solo será posible si productores y consumidores tienen un mínimo de sensibilidad para valores como la equidad, la solidaridad, la paz o la justicia.

Sin embargo, en medio de la situación actual podemos encontrar algunas excepciones. Por un Iado, la política de Responsabilidad Social Corporativa se ha convertido en una práctica habitual en las empresas en los últimos años. Por otro lado, existen una serie de colectivos que, en muchas ocasiones, pasan desapercibidos. Es el caso de la denominada economía social (p.ej.: ONGs, empresas sociales como La Fageda: www.fageda.com; Muhamad, 2008), de la economía del bien común (Felber, 2012), de la economía de comunión (www.edc-online.org), o la Banca ética (p.ej.: www.triodos.es). Estas iniciativas sociales están basadas en valores como solidaridad, confianza, responsabilidad y dignidad de la persona humana. Todas ellas nos hacen pensar que el cambio de actitud en el modelo empresarial se empieza a producir. También en los consumidores aparecen nuevas formas de consumir basadas en la responsabilidad y en la opción de compartir en lugar de poseer (p.ej.: comercio justo, comercio responsable o consumo colaborativo: www.consumocolaborativo.com). Según De Margarida (2013), en la actualidad, estas singularidades están adquiriendo una importancia considerable, ya que logran tanto fomentar el empleo como generar riqueza económica y social, constituyendo así un nuevo pilar sobre el que pueden apoyarse los Estados, las empresas y los ciudadanos para lograr cambiar el sistema económico y financiero que nos ha llevado al hundimiento del estado del bienestar.

También, en el mundo universitario, se ha incrementado notablemente en los últimos años el fomento de la responsabilidad, la solidaridad, la sostenibilidad y la cooperación al desarrollo. Universidades en todo el mundo se han comprometido a redefinir y reorientar su enseñanza, aprendizaje, investigación y gestión hacia la sostenibilidad (Cortese, 2003; Wright, 2004; Lozano, 2006; Lozano, 2010). Se han integrado cursos especializados e interdisciplinarios en los curriculum (p.ej.: Junyent y Geli, 2007; Sleurs, 2008) y las competencias han sido definidas en el marco de la sostenibilidad (Roorda, 2001; De Haan, 2006; Barth et al., 2007; Sleurs, 2008).

Como ejemplo de ello, citamos a continuación algunas acciones llevadas a cabo recientemente en el ámbito de la universidad española:

- La Estrategia de Cooperación Universitaria al Desarrollo, aprobada por la CRUE en septiembre de 2000 fue sin duda el punto de partida del trabajo de las universidades en esta nueva dirección y refleja la importancia de este aspecto en la universidad (CRUE, 2000).

- El Plan Director de la Cooperación Española 2005-2008 incorpora a la Universidad como Agente de la Cooperación al Desarrollo y la define como un ámbito privilegiado por dos motivos: como institución, por la posibilidad de desarrollar proyectos de cooperación al desarrollo y, por otro lado, por la sensibilización y educación, ya que puede transmitir valores solidarios a los jóvenes que tienen un papel social en el futuro (Min.As.Ext. y Coop, 2005). 
- Del 29 al 31 de octubre de 2012 se celebraron las I Jornadas sobre Universidad y Educación para el desarrollo en la Universidad Pública de Navarra, donde se ponía de manifiesto que la Educación para el Desarrollo (EpD) en la Universidad va cobrando cada vez mayor relevancia y va consolidándose.

- Del 24 al 26 de abril de 2013 se celebró el VI Congreso Universidad y Cooperación al Desarrollo en Valencia, que tuvo como objetivo analizar el papel de las Universidades en la promoción del Desarrollo Humano.

- La Estrategia Universidad 2015 convierte la responsabilidad social en el mundo universitario en un eje estratégico (Ministerio de Educación, 2011) y aparecen las primeras memorias de sostenibilidad y el compromiso con aspectos medioambientales, sociales y económicos.

- Existen Oficinas para la Cooperación al Desarrollo y Oficina Verde en casi todas las universidades españolas.

A pesar de todos estos esfuerzos, existen diferentes barreras tanto a nivel institucional, como a nivel individual para alcanzar la sostenibilidad y para incorporarla a las universidades. Por ejemplo, la dificultad de coordinación de varias iniciativas, la posible pérdida de poder de algunos agentes, la resistencia al cambio por temor a la dificultad, las limitaciones de las soluciones tecnológicas, las limitaciones de la información o el balance entre derechos individuales y universales (Lozano, 2006; Velázquez et al., 2006; Sibbel, 2009; Ceulemans et al., 2011). Dichas barreras provocan que las universidades estén lejos de tener una orientación real hacia la sostenibilidad (Wals y Jickling, 2002; Sterling, 2004; Sterling y Scott, 2008). Por lo que el desarrollo sostenible todavía es una idea innovadora en la mayoría de universidades (Lozano, 2006). El diseño del currículo y el temario de las asignaturas están más centrados en la transmisión de conocimientos que en la facilitación de espacios de crítica, innovación y aprendizaje creativo, sin que se plantee a los alumnos una alternativa al modelo actual, ni se les presenten las actuales iniciativas empresariales, universitarias y sociales.

De todo lo dicho anteriormente, podemos concluir que en la Universidad los estudiantes deben, no sólo adquirir una serie de competencias como trabajo en equipo o capacidad crítica y autocrítica; sino también competencias relacionadas con la sensibilidad social. Formar estudiantes que quieran contribuir a mejorar la sociedad se puede lograr desde dos perspectivas: (1) la universidad como institución y (2) desde el aula, por medio del profesorado. La primera opción está más desarrollada a tenor de las acciones que se vienen desarrollando desde hace más de una década (algunas de ellas, comentadas anteriormente). Sin embargo, convendría articular mecanismos para promover esta necesidad a nivel de profesorado y contar con programas de formación y motivación del profesorado. Pero la segunda opción se realiza en menor medida.

Existen numerosas maneras de sensibilizar a los estudiantes en temas sociales. Además, dicha sensibilización se puede despertar desde muchas asignaturas. No se trata de impartir conocimientos al alumnado, sino de concienciarlos sobre la trascendencia de ciertos aspectos sociales, tarea más complicada. Precisamente, el objetivo de este trabajo es contribuir a la introducción de la sensibilización social de los estudiantes universitarios en el aula. Para ello, presentamos un ejemplo de dos prácticas docentes realizadas en el marco de la enseñanza de administración y dirección de empresas. Se trata este de un marco de interés, ya que algunos de los alumnos que cursan estos estudios serán los futuros responsables de tomar muchas decisiones que afectarán al bienestar de las personas. En concreto, las prácticas se desarrollan en la asignatura de Organización de la Producción en $3^{\circ}$ de Ingeniería Electrónica Industrial y Automática, en la Universidad de La Rioja. 
El trabajo se estructura de la siguiente manera: en el apartado 2, se presenta el marco teórico de referencia sobre la temática de la sensibilización social de los estudiantes universitarios, así como una revisión de la literatura. En el apartado 3, se explican detalladamente las prácticas llevadas a cabo con los estudiantes. En el apartado 4 se presentan y discuten los resultados de las prácticas y de una encuesta hecha a los estudiantes, así como sus notas. Finalmente, el apartado 5, dedicado a conclusiones, se centra en las contribuciones del trabajo, las limitaciones y las mejoras futuras.

\section{SENSIBILIZACIÓN SOCIAL DE LOS ESTUDIANTES UNIVERSITARIOS}

Un debate existente en la literatura es si la sostenibilidad, la ética en los negocios o la Responsabilidad Social Corporativa deben enseñarse en asignaturas independientes o debe integrarse en el núcleo de las asignaturas de empresa (Christensen et al., 2007). Las asignaturas específicas permiten a los estudiantes explorar la esencia de los conceptos propios de sostenibilidad en detalle, pero el resultado puede ser que los estudiantes entiendan la sostenibilidad como un tema separado, desconectado de la estrategia empresarial, las finanzas, el marketing, los recursos humanos y la economía (Stubbs y Cocklin, 2008).

Esto puede estar pasando en la Universidad española, en la que numerosos Grados han incluido asignaturas específicas sobre organizaciones no lucrativas o Cooperación al Desarrollo. Asimismo, se han implantado Máster relacionados con la Cooperación al Desarrollo. Sin embargo, no en todas las asignaturas se persigue transmitir estos valores a los estudiantes.

Hay que tener en cuenta que los procesos de aprendizaje que permiten cambios transformadores dependen en gran medida del personal académico y de su capacidad y voluntad de apoyar este tipo de procesos (Hegarty, 2008). Esto implica que, aunque las universidades ayuden creando estructuras estables que contribuyen a mejorar, reforzar y diseñar actividades, es necesario el apoyo del profesorado para conseguir que el trabajo que intentan hacer las universidades llegue también a las aulas. Los temas que suponen una implicación de índole social deben ser tratados en las aulas como parte del currículo de los estudiantes. Por ello, el papel del profesorado es de vital importancia (Cerrillo Martín, 2003; Arias Careaga, 2008). En este sentido, es necesario reflexionar sobre el rol del profesor para que este sea, no sólo el mediador del saber académico, sino también de la vida y la sociedad (Fernández, 1991).

Si nos centramos en las prácticas docentes para la sensibilización social de los estudiantes universitarios, encontramos que, en los últimos años, se viene trabajando en la 'Educación para el Desarrollo Sostenible (EDS)', la 'Educación Solidaria', la 'Educación para la Cooperación al Desarrollo' o el 'Aprendizaje-Servicio'.

En la literatura científica se desarrolla sobre todo la primera. Ésta, requiere enseñanza participativa y métodos de enseñanza que motiven para un cambio de comportamiento y lleve a la acción para un desarrollo sostenible. La Década de Educación para el Desarrollo Sostenible de las Naciones Unidas (2005-2014) exige una integración completa de los problemas de sostenibilidad a todos los niveles de la educación, a través de un enfoque interdisciplinario y transdisciplinario y con un claro enfoque en los valores. La cultura de la sostenibilidad comporta la adopción fuerte de los valores morales de la justicia y la solidaridad, por lo que es fundamentalmente un proyecto moral (Ortega y Mínguez, 1998).

Debido a esta necesidad, recientemente se están publicando numerosos trabajos en torno a este ítem. Algunos trabajos han documentado y analizado cuáles son las competencias propias de sostenibilidad que deben conseguir los estudiantes (Roorda, 2001; de Haan, 2006; Sterling y Thomas, 2006; Barth et al., 2007; Sipos et al., 2008). 
Numerosos casos de estudios analizan estas competencias en educación sostenible en una universidad concreta (Lambrechts et al., 2012), incluso con la utilización de un programa informático (Lozano, 2010). Otros se centran en evaluar e informar el esfuerzo de los alumnos, con nuevos indicadores y herramientas de evaluación (Roorda, 2002; Behrens y Müller-Christ, 2005; Lozano y Peattie, 2011). Por último, existen métodos y técnicas para enseñar y evaluar programas y competencias para el desarrollo sostenible (Steiner y Posch, 2006; Junyent y Geli, 2007; Ceulemans y De Prins, 2010; Yarime y Tanaka, 2012).

Algunos trabajos proponen diferentes tipos de actividades docentes que ayudan a conseguir conocimientos, habilidades y actitudes para la sostenibilidad (Stubbs y Schapper, 2011). Sin embargo, son pocos los trabajos que proponen actividades docentes concretas que se pueden llevar a cabo para que los estudiantes alcancen las competencias de sostenibilidad. Algunos de ellos analizan los conocimientos y actitudes ante el turismo ético (García Sánchez y Green, 2013), realizan un código ético con los alumnos (Bernardo y Tarí, 2013) o proponen una empresa social (Queiruga et al., 2011).

En los últimos años empiezan a surgir en el ámbito de la sostenibilidad 'oportunidades de aprendizaje en el mundo real' y, poco a poco, empiezan a ser trasladadas estas experiencias a la literatura. A través de estos ejemplos se consigue analizar y evaluar el grado en el que se logra alcanzar las competencias en sostenibilidad (Brundiers et al., 2010).

Hasta ahora hemos visto la necesidad de educar en valores para la sostenibilidad y cómo se ha llevado a cabo por distintos autores. De todas las formas posibles de llevar la sensibilización social al aula, nosotros hemos elegido relacionar los valores con el contenido didáctico (Parra Ortiz, 2003), debido a que en algunas asignaturas prácticamente no se transmiten valores y por la innovación y necesidad que supone llevar al aula estos valores. Para ello, proponemos dos prácticas docentes de contenido social que pueden integrarse dentro del resto del contenido de la asignatura.

\section{PRÁCTICAS DOCENTES PARA LA SENSIBILIZACIÓN SOCIAL DE LOS ESTUDIANTES UNIVERSITARIOS}

A continuación se muestran las dos prácticas docentes que preparamos los autores de este trabajo para la asignatura de Organización de la Producción en $3^{\circ}$ de Ingeniería Electrónica Industrial y Automática, en la Universidad de La Rioja, el curso académico 2012-2013.

La idea de elaborar una práctica sobre la reutilización de residuos de aparatos eléctricos y electrónicos, surgió a partir de una línea de investigación de los autores de este trabajo sobre la gestión de estos residuos (p.e. Queiruga et al., 2012). Para la elaboración de la práctica del banco de alimentos nos basamos en nuestra experiencia docente en asignaturas de dirección de la producción y logística y gestión de almacenes.

Para trasladarlo al ámbito social, planteamos el mismo tipo de ejercicios, pero a realizar sobre una organización no lucrativa real, en lugar de una empresa lucrativa ficticia.

El contenido de las prácticas se describe a continuación: 


\section{Práctica 1: Logística INVERSA de lOS RESIDUOS de aparatos ElÉCtricos} Y ELECTRÓNICOS: LA REUTILIZACIÓN VS. EL RECICLAJE.

\section{Introducción:}

La revisión de la Directiva Europea sobre Residuos de Aparatos Eléctricos y Electrónicos (RAEE) hace hincapié en el fomento de la reutilización. En el año 2016 se deberán recoger $45 \dagger$ de RAEE por cada $100 \dagger$ de aparatos puestos en el mercado. Por tanto, a partir de este año, se deberá no sólo reciclar, sino reutilizar y reciclar.

\section{La práctica consiste en:}

- Hacer un esquema del sistema de logística inversa de los RAEE, en el que aparezcan los agentes involucrados en la gestión de estos residuos en tu Comunidad Autónoma.

- Calcular los costes de la logística inversa de los RAEE.

- Averiguar qué es más favorable para la sociedad en su conjunto: sólo reciclar, o reutilizar y reciclar. Para ello, debemos tener en cuenta factores económicos (costes), sociales (acceso de personas necesitadas a electrodomésticos baratos, creación de empleo...) y medioambientales (reducción de residuos).

Para resolver la práctica, te puede ser útil esta información:

\section{Agentes económicos involucrados:}

- Fabricantes (Considerar dos. Por ejemplo: Fagor, Balay...)

- Distribuidores (Considerar cuatro de La Rioja. Por ejemplo: MediaMarkt, Carrefour, Eroski,...)

- Puntos limpios (ver localización, por ejemplo en la web de la Fundación ECOLEC o en la Consejería de Medioambiente)

- Plantas de reciclaje específicas para RAEE: Indumetal Recycling (Bilbao); Irsa Office (Madrid); Electrorecycling (Barcelona); Recilec (Sevilla); Recytel (Madrid)

- Empresas que reparan RAEE. Ejemplos: Revertia: planta gestora de RAEE para su reutilización (revertia.com); Ekorrepara: Centro para la reutilización Red Social Koopera (www.ekorrepara.com)

- Sistemas Integrados de Gestión (SIG): ECOLEC, ECOTIC, SIGCLIMA, AMBILAMP, ECOLUM, ECORAEES, TRAGAMOVIL, ECOFIMATICA, ECOPILAS

- Plataforma informática: OfiRAEE (aquí encontráis el acceso a los SIG)

\section{Otras fuentes de información sobre el tema de RAEE:}

- Registro de Aparatos Eléctricos y Electrónicos puestos en el mercado por los fabricantes:

www.minetur.gob.es/industria/RAEE/Paginas/Index.aspx

- Información sobre la Directiva Europea, noticias, Jornadas: Cátedra RELEC www.relec.es/relec/

- Características del sistema de gestión español. Artículo: Queiruga, D.; González Benito, J.; Lannelongue, G. (2012): Evolution of the electronic waste management system in Spain. Journal of Cleaner Production 24, 56-65. 
Práctica 2: Gestión de almacenes en el Banco de Alimentos

- Busca información acerca del Banco de Alimentos (qué es, qué hace, dónde está...).

- ¿ ¿Cuáles creen que son los objetivos más importantes para el Banco de Alimentos y las ONGs con las que colabora?

- Haz un esquema del sistema logístico del flujo de alimentos en el que aparezcan todos los agentes involucrados, desde la obtención de los alimentos hasta las familias (te puede ayudar la Memoria del Banco de Alimentos).

- ¿ ¿Cuál es el número de familias necesitadas de alimentos en La Rioja? (Algunas fuentes de información: INE, Cáritas, periódicos con información sobre el número de familias en las que los dos miembros están en paro).

- Calcula los costes de transporte, almacén y mano de obra del Banco de Alimentos.

- Utilizando las fórmulas de gestión de inventarios, ¿ Cada cuánto tiempo debería hacer una campaña de recogida de alimentos? ¿Cada cuánto tiempo debería hacer un envío a las ONGs?

- ¿ ¿Qué política de inventarios crees que sería la más acertada? (revisar el almacén periódicamente, o constantemente, mantener un nivel mínimo, etc.)

- ¿ ¿Tendría sentido la automatización de los almacenes? ¿ Cuál sería el coste de la automatización? ¿Qué grado de automatización sería el más apropiado? (por ejemplo: sólo carretillas eléctricas, o hasta robots que preparasen los pedidos?) (fuente: mecalux.es)

- $\quad$ ¿Qué pasos tendrías que seguir si quisieras hacer una campaña de recogida de alimentos en la Universidad de La Rioja para llevárselos al Banco de Alimentos? ¿Cuál crees que sería el coste que te supondría? ¿Qué cantidad de alimentos crees que obtendrías? ¿Cuántas familias se beneficiarían y durante cuánto tiempo?

Las dos prácticas fueron realizadas por los 24 alumnos matriculados en la asignatura, en el aula de informática. Los alumnos comenzaron las prácticas en horario de clase, para facilitar el comienzo de la actividad, su comprensión y poder responder posibles dudas.

Se trata de prácticas que se pueden realizar independientemente del número de alumnos, individualmente $o$ en grupos pequeños. En nuestro caso, elegimos que formaran grupos de dos o tres alumnos para que pudieran ayudarse mutuamente. Unos grupos trabajaban más rápidamente que otros. Si a algún alumno le surgía alguna pregunta puntual acerca de dónde encontrar información, qué fórmula de gestión de almacenes era la apropiada o qué función de Excel utilizar, se ayudaban unos a otros. Las terminaron en casa con un plazo de una semana. Este tiempo fue suficiente y en ningún caso sugirieron que necesitaran más. Para responder las preguntas propuestas utilizaron la información contenida en internet y los conocimientos teóricos recibidos en clase. Las entregaron en el aula virtual e hicieron una exposición oral de 10 minutos unos días después de la corrección de las prácticas.

Las prácticas expuestas suponían:

- Práctica 1 (Reutilización de RAEE): Entrega trabajo: 0,25\% de la nota final Exposición oral: $0,25 \%$ de la nota final

- Práctica 2 (Banco de Alimentos): Entrega trabajo: 0,25\% de la nota final Exposición oral: $0,25 \%$ de la nota final 


\section{RESULTADOS Y DISCUSIÓN}

Un aspecto muy positivo de la realización de estas prácticas es que no tienen un resultado predeterminado, ni una solución única a la que tienen que llegar. En el caso de la práctica 1, los estudiantes tienen que elegir las fábricas, elegir las plantas de reutilización y reciclaje, elegir los camiones que utilizarán para el transporte y calcular los Residuos de Aparatos Eléctricos y Electrónicos recogidos, explicando qué criterios siguen para cada elección. En el caso de la práctica 2, los estudiantes tienen que buscar información como familias necesitadas en la Rioja, ONGs con las que trabajan, su situación o costes de transporte. Aunque en un primer momento algunos alumnos parecían desconcertados con la peculiaridad de buscar ellos mismos la información para la resolución de los ejercicios, pronto resultó ser un elemento motivador para todos. También es positivo el hecho de que no pueden encontrar en internet una solución del trabajo. Para evitar que en años sucesivos los estudiantes puedan copiar los trabajos, se pueden realizar las mismas actividades, pero basadas en otras Comunidades Autónomas distintas.

En toda la asignatura, los estudiantes respondieron muy bien y obtuvieron buenos resultados. En estas dos prácticas, las notas también fueron muy satisfactorias (ver Figura 1).

\section{Figura 1: NotAS de LOS ALUMNOS EN LAS PRÁCticaS}

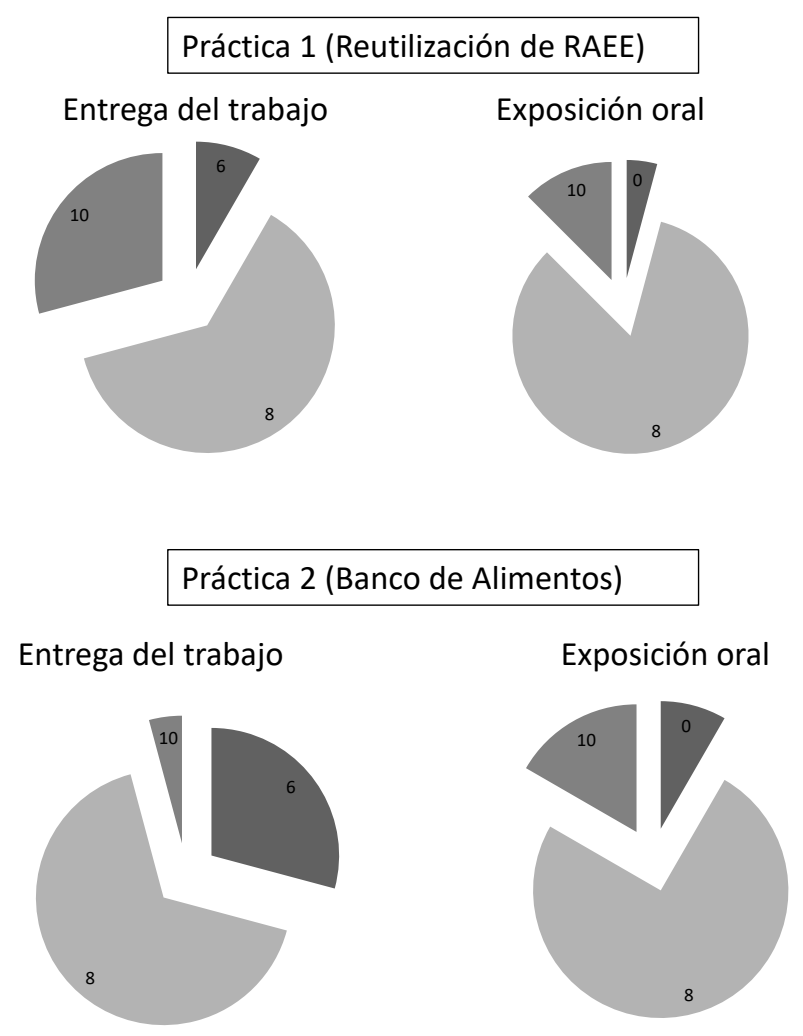

Medir los resultados de este tipo de actividades no resulta tan sencillo como medir la adquisición de conocimientos. Esto es debido a que la Educación para el Desarrollo Sostenible debe incluir distintas formas de creación de capacidades que incluyan 'cabeza, manos y corazón' (Bloom et al., 1964; Orr, 2002; Sipos et al., 2008) y saber si los 
estudiantes han alcanzado estas capacidades es muy complicado. Un estudiante que haya realizado las prácticas puede haber reflexionado sobre la necesidad de contribuir a mejorar la sociedad, pero cuando termina el curso, olvidarse de lo que había pensado. Otro compañero podría haber sido indiferente al aspecto social de la práctica, pero recordarlo después de unos años.

A pesar de la dificultad de la medición, hicimos un intento de valorar la repercusión en los estudiantes. Desde la implantación del Espacio Europeo, se vienen realizando encuestas a los estudiantes para conocer qué prácticas docentes son las que más les motivan. En nuestra asignatura también realizamos una encuesta de este tipo, en la que los estudiantes valoraban las prácticas de 1 a 5 y respondían otras preguntas afirmativa o negativamente. En este caso el formato de la encuesta es similar a las encuestas de satisfacción de los estudiantes con la actividad docente del profesor. Algunas de las preguntas eran generales sobre la asignatura, mientras otras iban encaminadas a conocer si consideraban que las prácticas realizadas eran apropiadas para la asignatura y a conocer si les habían hecho reflexionar sobre las necesidades sociales. En la Figura 2 se muestra un extracto de la encuesta con las preguntas referidas a las prácticas de este artículo.

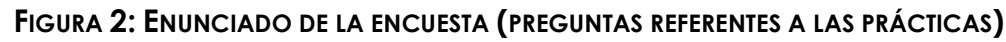

\section{ENCUESTA DE ACTIVIDADES DOCENTES}

Pregunta 1.- Valora de 1 a 5 las siguientes actividades docentes de la asignatura Organización de la Producción según si consideras que son apropiadas y que has aprendido con ellas (1: muy poco apropiada; 5: muy apropiada):

- Exposición oral de las prácticas en clase

- Trabajo en grupo

- Práctica de informática sobre reutilización de RAEE

- Práctica sobre el banco de alimentos

$$
\begin{aligned}
& {[1-2-3-4-5]} \\
& {[1-2-3-4-5]} \\
& {[1-2-3-4-5]} \\
& {[1-2-3-4-5]}
\end{aligned}
$$

Pregunta 2.- ¿Alguna actividad de clase te ha hecho reflexionar sobre las necesidades sociales y la necesidad de colaborar?

Sí. ¿̇Cuál?

No.

Pregunta 3.- ¿ُParticipas en alguna ONG como voluntario, socio o dando donativo?

Sí. ¿̇Con qué frecuencia?.

No. ¿̇Tienes pensado participar en el futuro?

La encuesta fue contestada por 23 estudiantes de un total de 24 matriculados y ofrecieron los siguientes resultados:

$1 l^{a}$ pregunta: Los resultados de esta pregunta se presentan en la Figura 3.

- Exposición oral de las prácticas en clase: Ningún alumno contestó que no fuera apropiado. 
- Trabajo en grupo: Ningún alumno contestó que no fuera apropiado.

- Práctica de reutilización de RAEE: Sólo un $4 \%$ de los estudiantes consideraron que no fueron apropiadas.

- Práctica del Banco de Alimentos: sólo un 9\% de los estudiantes consideraron que no fueron apropiadas.

2a pregunta: Las respuestas fueron:

- Sí (83\% de los alumnos). El 57\% de los alumnos encuestados especificó que fue la práctica del Banco de Alimentos la que le hizo reflexionar.

- No $(0 \%)$

- En blanco (17\% de los alumnos)

3a pregunta: Las respuestas fueron:

- Sí (0\%)

- No (100\%). El 43,4\% de los alumnos encuestados indicó que le gustaría participar en el futuro en alguna ONG.

- En blanco (0\%)

Figura 3: Resultados de LAS Respuestas a LA PREgUnta 1

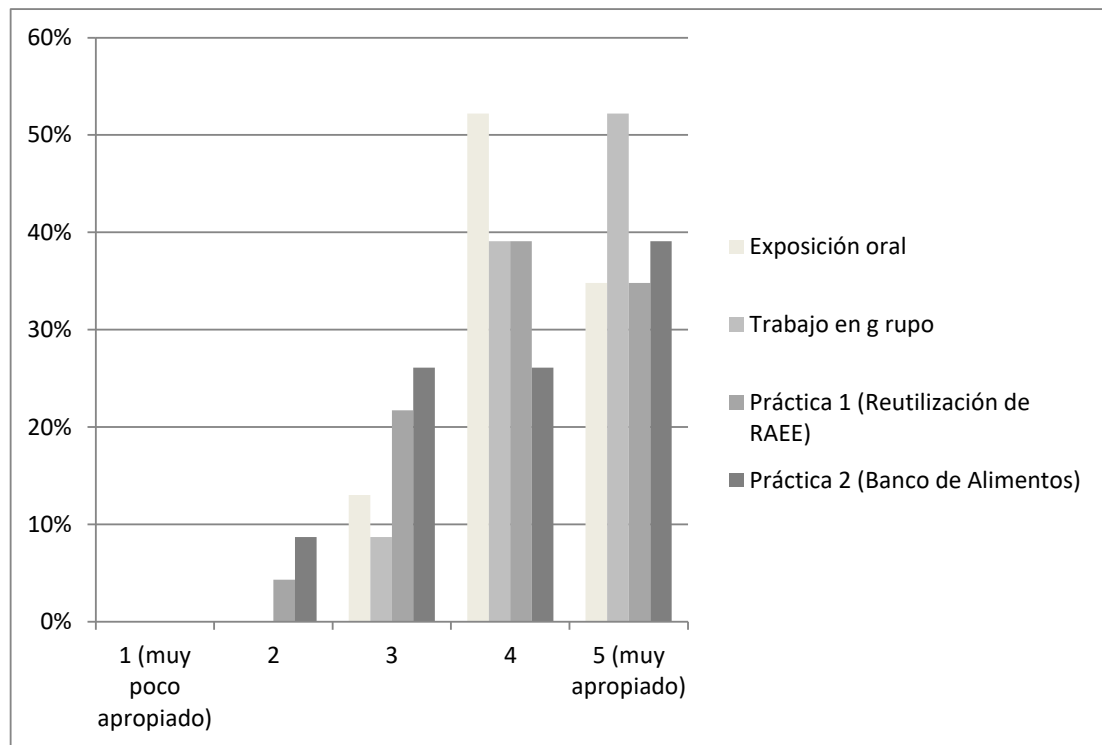

No podemos esperar que unas prácticas hechas en una sola asignatura cambien la vida, las actitudes y las motivaciones de los alumnos ante las necesidades humanas de una manera radical. Ni siquiera podemos esperar que la sensibilización llegue a todos los alumnos, porque va a depender mucho de sus actitudes iniciales y su vida familiar y educativa. Hay que tener en cuenta que en la Universidad puede ser difícil inculcar unos valores que el alumno no haya percibido previamente. Este cambio suele producirse cuando la ayuda se transforma en acción. Por este motivo, el objetivo de este trabajo era aportar un granito de arena a la gran misión de hacer el mundo más solidario. El hecho de que la mayoría de alumnos haya reflexionado sobre las necesidades sociales y la necesidad de colaborar nos hace pensar que hemos conseguido el objetivo que nos planteamos. Sin embargo, para conseguir la sensibilidad social sería recomendable realizar este tipo de actividades en el resto de asignaturas y a nivel de Facultades. 
Se citan a continuación algunos ejemplos para poder incorporar la sensibilización en distintas asignaturas:

- En Contabilidad, se podría trabajar con el balance de una empresa adherida a la Economía del Bien Común y analizar las diferencias con el resto de empresas.

- En Dirección Estratégica, se podrían ver vídeos y lecturas sobre todas las alternativas empresariales planteadas en la introducción de este trabajo y debatir sobre: si es una alternativa real al modelo actual y las diferencias de objetivos teniendo en cuenta a los distintos grupos de interés.

- En Economía de la Empresa, podrían analizar las funciones de la empresa en una empresa social (Queiruga et al., 2011).

- En Dirección de Recursos Humanos, podrían plantearse cómo se realiza la selección de personal en una ONG de acción social.

Para poder añadir la sensibilización social en varias asignaturas, sería recomendable repasar las competencias transversales de todas asignaturas de los títulos de Grado desde la Facultad, desde la perspectiva del compromiso social.

\section{CONCLUSIONES}

La contribución de este trabajo se ve reflejada en los aspectos positivos que reportan a los estudiantes:

- Resultan muy motivadoras porque tratan sobre la realidad de su entorno, no sobre empresas ficticias.

- Ser conscientes de la realidad social. En concreto, con las dos prácticas planteadas en este trabajo, de las necesidades de alimento y de cuidado del medioambiente. Esto ayuda a desarrollar en ellos la humanidad, al ser capaz de detectar los problemas sociales y plantearse cómo se pueden solucionar.

- Reflexionar sobre las repercusiones de sus actos, tanto a corto plazo (p.ej.: el banco de alimentos) como a largo plazo (p.ej.: con el cuidado del medio ambiente).

- Valorar el esfuerzo de las personas que trabajan en la organización y reflexionar sobre el enorme potencial que tienen y cómo podrían contribuir ellos a mejorar la situación social en España.

- Conocer las iniciativas y avances que se están produciendo en el mundo empresarial, en concreto, los numerosos proyectos y empresas sociales que se están creando en los últimos años.

- Aunque nuestro objetivo directo no era preparar a los estudiantes para el mundo laboral, y teniendo en cuenta que las competencias más valoradas en los economistas por las empresas son las relativas a los 'valores' (Periáñez et al., 2010), indirectamente hemos conseguido también ayudar a los estudiantes a prepararse para un puesto de trabajo.

Un aspecto positivo de estas prácticas para los profesores es que se integran dentro de la asignatura, por lo que no suponen una dedicación de tiempo extra, sino que se utilizan como un recurso didáctico más en el desarrollo del temario de la asignatura.

La puesta en práctica de estas actividades en el aula nos ha hecho ver algunos aspectos que se pueden mejorar en próximos cursos. Las mejoras no se refieren al desarrollo de la práctica en sí misma, ya que hemos encontrado que tiene la misma manera de resolución que cualquier otro ejercicio sobre una empresa ficticia. En 
cuanto a la evaluación, si solo se evaluase el resultado de los ejercicios y la exposición oral, no habría diferencia con otros ejercicios. Sin embargo, una mejora que debemos introducir es hacer un esfuerzo por evaluar también la sensibilización social mediante instrumentos de medición de competencias. Por otro lado, encontramos bastante pasividad en los estudiantes en cuanto a la iniciativa de poner en marcha alguna actividad social: se les preguntó qué tendrían que hacer para llevar a cabo una recogida de alimentos, pero en ningún caso tuvieron la iniciativa de llevarla a cabo. Por tanto, estas mejoras se basan en intentar que la sensibilización social llegue más intensamente a los estudiantes y en evaluarlo.

Desde nuestro punto de vista, la Universidad es el lugar ideal para que surjan nuevas iniciativas entre estudiantes. Por ejemplo, para que ellos mismos hagan una campaña de recogida y reparto de alimentos o ropa, planteándose cada uno de qué manera pueden ayudar.

\section{BIBLIOGRAFÍA}

ARIAS CaReaga, S. [2008]: La cooperación universitaria al desarrollo. Un desafío permanente, Taban que. Revista Pedagógica, vol. 20: 11-26.

Barth, M., GodemanN, J., Riechman, M. y Stoltenberg, U. [2007]: Developing key competences for sustainable development in higher education, International Journal of Sustainability in Higher Education, vol. 8, núm. 4: 416-430.

BEHRENS, B. Y MüLLER-CHRIST, G. [2005]: Innovative Sustainability Monitoring and Reporting for Universities. Conference on the International Launch in Higher Education: Conference Proceedings: Committing Universities to Sustainable Development. Graz (Austria), abril: 239-248.

BERMÚDEZ LÓPEZ, F. [2010]: De la crisis a la esperanza. Sepha.

BERNARDO, M. Y TARÍ, J.J. [2013]: Propuesta de código ético para la asignatura de Gestión de la Calidad: comparativa interuniversitaria. Revista Educade, núm. 4: 111123.

BLOOM, B.S., MASIA, B.B. Y KRATHWOHL, D.R. [1964]: Taxonomy of Educational Objetives. David McKay, New York, NY.

BRUNDIERS, K., WIEK, A. Y REDMAN, CH.L. [2010]: Real-World learning opportunities in sustainability: from classroom into the real world, International Journal of Sustainability in Higher Education vol. 11, núm. 4: 308-324.

COLOM CAÑELlas, A. J. [2000]: El desarrollo sostenible y la educación para el desarrollo. Ediciones Octaedro, Madrid.

Cerrillo Martín, M.R. [2003]: Educar en valores, misión del profesor, Tendencias pedagógicas, vol. 8: 59-68.

Ceulemans, K., De Prins, M., Cappuyns, V. y De Coninck, W. [2011]: Integration of sustainable development in higher education's curricula of applied economics: large scale assessments, integration strategies and barriers, Journal of Management and Organization, vol. 17: 621-640.

Ceulemans, K. Y De PRINS, M. [2010]: Teacher's manual and method for SD integration in curricula, Journal of Cleaner Production, vol. 18: 645-651.

Christensen, L.J., PeirCe, E., Hartman, L.P., Hoffman, W.M. y CARRIER, J. [2007]: Ethics, CSR, and sustainability education in the Financial Times top 50 global business schools: baseline data and future research directions, Journal of Business Ethics, vol. 73, núm. 4: 347-368.

CRUE (CONFERENCIA DE RECTORES DE LAS UNIVERSIDADES ESPAÑOLAS) [2000]: Estrategia de Cooperación Universitaria al Desarrollo, http://www.ulpgc.es/hege/almacen/download/7060/7060492/crue_estrategi a_cooperacion_universitaria_desarrollo.pdf 
CORTESE, A.D. [2003]: The critical role of higher education in creating a sustainable future, Planning for Higher Education, vol. 31, núm. 3: 15-22.

DE HAAN, G. [2006]: The BLK 21 programe in Germany: a Gestaltungskompetenz-based model for education for sustainable development, Environmental Education Research, vol. 12, núm. 1: 19-32.

De MARGaridA, J.C. [2013]: Nuevo modelo económico basado en la economía social,http://www.castillayleoneconomica.es/blogs/economia-confundamento/nuevo-modelo-econ\%C3\%B3mico-basado-en-laeconom\%C3\%AD-social.

escámez SÁnchez, J., García lópez, R. y Pérez Pérez, C. [2003]: La educación moral ante el reto de la pobreza, Teoría de la Educación, vol. 15: 185-212.

FELBER, CH. [2012]: La economía del bien común, Deusto, Madrid.

FERNÁNDEZ, F. [1991]: La sociología y los profesores, Cuadernos de pedagogía, 190: 2021.

García SÁnCheZ, M.R. y Green, C.G. [2013]: Conocimiento y actitudes de los alumnos universitarios sobre sostenibilidad en el turismo. Workshop ACEDEDOT. Sevilla, abril.

GÓmEZ-HeRAS, J.M.G. [1997]: "EL problema de una ética del medio ambiente", en J.M.G Gómez Heras (Coord.) Ética del medio ambiente. Problema, perspectivas, historia, Tecnos, 17-71, Madrid.

HEGARTY, K. [2008]: Shaping the self to sustain the other. Mapping impacts of academic identity in education for sustainability, Environmental Education Research, vol. 14: 681-692.

JUNYENT, M. Y GeLl, A.M. [2007]: The ACES Network: Curriculum Greening of Higher Education. Good Practices in Education for Sustainable Development. Teacher Education Institutions. UNESCO-Education for Sustainable Development in Action. Good Practices, $n^{\circ} 1-2007$.

LambrechtS, W., Mulà, I., Ceulemans, K., Molderez, I. y Gaeremynck, V. [2012]: The integration of competences for sustainable development in higher education: an analysis of bachelor programs in management, Journal of Cleaner Production, vol. 48: 65-73.

LOZANO, R. [2006]: Incorporation and institutionalization of SD into universities: breaking through barriers to change, Journal of Cleaner Production, vol. 14: 787-796.

LOZANO, R. [2010]: Diffusion of sustainable development in universities' curricula: an empirical example from Cardiff University, Journal of Cleaner Production, vol. 18: 637-644.

LOZANO, R. y PEATTIE, K. [2011]: Assessing Cardiff University's curricula contribution to SD using the STAUCH(RTM) system, Journal of Education for Sustainable Development, vol. 5: 115-128.

MÉndez GarCíA, J.M. [2012]: ¿Crisis económica o crisis de valores? Sepha, Madrid.

MINISTERIO DE ASUNTOS EXTERIORES Y COOPERACIÓN [2005]: Plan Director de la Cooperación Española,

www.aecid.es/galerias/publicaciones/descargas/Plan_Director0508_Esp.pdf

MINISTERIO De EDUCACIÓN [2011]: La Responsabilidad Social de la Universidad y el Desarrollo Sostenible. Secretaría General Técnica.

MORENO, J. [2013]: Davos: ¿̇crisis del capitalismo o crisis ética?, http://www.elmundo.com.ve/firmas/jose-i--moreno-leon/davos--̇̇crisis-delcapitalismo-o-crisis-etica-.aspx

MUHAMAD, Y. [2008]: Un mundo sin pobreza. Paidós, Madrid.

ORTEGA P. Y MíngUEZ, R. [1998]: Educación, cooperación y desarrollo, Revista española de pedagogía, vol. 211 , 457-482.

Orr, D.W. [2002]: Four challenges of sustainability. Conservation Biology, vol. 16, núm. 6: $1457-1460$. 
PARRA ORTIZ, J.M. [2003]: La Educación en valores y su práctica en el aula, Tendencias pedagógicas, vol. 8: 69-88.

Periáñez Cañadillas, I., luengo Valderrey, M.J., Pando García, J., De la Peña García, J.I. y VILLABA MerLo, F.J. [2010]: Competencias demandadas en los nuevos economistas. Revista de Educación en Contabilidad, Finanzas y Administración de Empresas (Educade) vol. 1: 59-77.

Queiruga, D., González Benito, J. y Lannelongue Nieto, G. [2011]: Actividades docentes para la sensibilización social de los estudiantes universitarios. II Workshop ACEDEDOT, Segovia, 4 abril.

Queiruga, D., GONZÁlez Benito, J. y LANnelongue Nieto, G. [2012]: Evolution of the electronic waste management system in Spain. Journal of Cleaner Production vol. 24: 5665.

ROORDA, N. [2001]: Auditing instrument for the integration of sustainability in higher education "AISHE", DHO, Amsterdam.

ROORDA, N. [2002]: Assessment and policy development of sustainability in higher education with AISHE. In: Filho, W.L. (Ed), Teaching Sustainability at Universities: Towards Curriculum Greening, Environmental Education, Communication and Sustainability. Peter Lang, Frankfurt.

ROVIRA, A. Y OLMOS, P. [2013]: La vida que mereces. Conecta, Madrid.

SÁNCHEZ CÁMARA, I.[ 2012]: Europa y sus Bárbaros. Rialp, Madrid.

SÁNCHEZ-QUIÑNONES, J. [2011]: Crisis económica y crisis de valores. El Confidencial, http://blogs.elconfidencial.com/economia/informacionprivilegiada/201 1/12/27/crisis-economica-y-crisis-de-valores-6444

SIBBEL, A. [2009]: Pathways towards sustainability through higher education. International Journal of Sustainability in Higher Education, vol. 10, $\mathrm{n}^{\circ} 1: 68-82$.

SIPOS, Y., BATTISTI, B. Y GRIMM, K. [2008]: Achieving transformative sustainability learning: engaging heads, hands and heart, International Journal of Sustainability in Higher Education, vol. 9, núm. 1: 68-86.

SLEURS, W. (ED.) [2008]: Competences for ESD (Education for Sustainable Development) Teachers. A Framework to Integrate ESD in the Curriculum of Teacher Training Institutes (Brussels).

SteINER, G. Y POSCH, A. [2006]: Higher education for sustainability by means of transdisciplinary case studies: an innovative approach for solving complex, real-world problems, Journal of Cleaner Production, vol. 14: 877-890.

STERLING, S. [2004]: Higher Education, sustainability, and the role of systemic learning. En: Higher Education and the Challenge of Sustainability: Problematics, Promise and Practice, 49-70. Kluwer Academic Publishers. Netherlands.

STERLING, S. Y SCOTT, W. [2008]: Higher education and ESD in England: a critical commentary on recent initiatives, Environmental Education Research, vol. 14, núm. 4: 386-398.

STERLING, S. Y THOMAS, I. [2006]: Education for sustainability: the role of capabilities in guiding university curricula, International Journal of Innovation and Sustainable Development, vol. 1, núm. 4: 349-370.

STUBBS, W. y COCKLIN, C. [2008]: Teaching sustainability to business students: shifting mindsets, International Journal of Sustainability in Higher Education, vol. 9, núm. 3: 206-221.

STUBBS, W. Y SCHAPPER, J. [2011]: Two approaches to curriculum development for educating for sustainability and CSR, International Journal of Sustainability in Higher Education, vol. 12, núm. 3: 259-268.

VelázQuez, L., Munguia N., Platt A. y TAdDel J. [2006]: Sustainable university: what can be the matter?, Journal of Cleaner Production, vol. 14: 810-819.

WALS, A.E.J. Y JICKLING, B. [2002]: Sustainability in higher education: from doublethink and newspeak to critical thinking and meaningful learning, Higher Education Policy, vol. 15: 121-131. 
WRIGHT, T. [2004]: The evolution of sustainability declarations in higher education. In: Corcoran, P.B., Wals, A. en (Eds.) Higher Education and the Challenge of Sustainability. Problematics, Promise and Practice. Kluwer Academic Publishers, Dordrecht/Boston/London/, 7-20.

YARIME, M. Y TANAKA, Y. [2012]: The Issues and Methodologies in Sustainability Assessment Tools for Higher Education Institutions. A Review of Recent Trends and Future Challenges, Journal of Education for Sustainable Development, vol. 6, núm.1: 63-77. 